\title{
Plasma extracellular vesicle protein content for diagnosis and prognosis of global cardiovascular disease
}

\author{
J. W. Wang • C. M. Gijsberts • A. Seneviratna • V. C. de Hoog • J. E. P. Vrijenhoek • \\ A. H. Schoneveld • M. Y. Chan - C. S. P. Lam • A. M. Richards • C. N. Lee • \\ A. Mosterd • S. K. Sze • L. Timmers • S. K. Lim • G. Pasterkamp • D. P. V. de Kleijn
}

Published online: 23 August 2013

(C) The Author(s) 2013. This article is published with open access at Springerlink.com

\begin{abstract}
Cardiovascular disease is a major public health problem worldwide. Its growing burden is particularly ominous in Asia, due to increasing rates of major risk factors such as diabetes, obesity and smoking. There is an urgent need for early identification and treatment of individuals at risk of adverse cardiovascular events. Plasma extracellular vesicle proteins are novel biomarkers that have been shown to be useful in the diagnosis, risk stratification and prognostication of patients with cardiovascular disease. Ongoing parallel
\end{abstract}

J. W. Wang $\cdot$ A. Seneviratna $\cdot$ C. N. Lee $\cdot$ D. P. V. de Kleijn Department of Surgery, National University Singapore (NUS), Singapore, Singapore

J. W. Wang • A. Seneviratna • M. Y. Chan • C. S. P. Lam •

A. M. Richards $\cdot$ D. P. V. de Kleijn $(\square)$

Cardiovascular Research Institute (CVRI), National University Heart Centre Singapore (NUHCS), National University Health System,

Singapore, Singapore

e-mail: surdvp@nus.edu.sg

C. M. Gijsberts • V. C. de Hoog • J. E. P. Vrijenhoek •

A. H. Schoneveld · L. Timmers · G. Pasterkamp • D. P. V. de Kleijn

Experimental Cardiology, Cardiology, UMC Utrecht,

the Netherlands

C. M. Gijsberts · J. E. P. Vrijenhoek • A. H. Schoneveld •

D. P. V. de Kleijn

Interuniversity Cardiology Institute of the Netherlands (ICIN),

Utrecht, the Netherlands

A. Mosterd

Meander Medical Center, Amersfoort, the Netherlands

S. K. Sze

School of Biological Sciences, Nanyang Technological University, Singapore, Singapore

\section{S. K. Lim}

Institute of Medical Biology, A*Star, Singapore biobank initiatives in European (the Netherlands) and Asian (Singapore) populations offer a unique opportunity to validate these biomarkers in diverse ethnic groups.

\section{Cardiovascular disease is a global problem}

Cardiovascular disease accounted for $30 \%$ of an estimated 58 million deaths globally from all causes in 2005 [1]. It is important to recognise that a substantial proportion of these deaths ( $46 \%$ ) occurred in individuals under 70 years of age, in their productive years of life; in fact, $79 \%$ of the disease burden attributed to cardiovascular disease is in this age group [2]. Moreover, between 2006 and 2015, deaths due to noncommunicable diseases (half of which are due to cardiovascular disease) are expected to increase by $17 \%$ [1]. This projected increase is most prominent in Asia due to a very large, ageing population, increasing exposure to a Westernised diet, and rapidly increasing rates of diabetes mellitus [3].

Early detection of patients at risk and early institution of effective treatment may help to ease the mortality and morbidity burden of cardiovascular disease. To achieve this, tools for early diagnosis and risk stratification of patients are urgently needed.

Ischaemic heart disease is a major cardiovascular cause of death worldwide. Improved early diagnosis in patients with chest pain in or before the emergency department would reduce the burden on the hospitals. Furthermore, in an ageing population with improved outcome after myocardial infarction, the number of patients with an elevated risk for a second cardiovascular event is increasing but identification of patients at high risk of recurrent events remains difficult. Increasing survival following acute coronary events also leads to more patients living to develop heart failure (HF). HF, the final common pathway of cardiac insults, is a serious syndrome 
due to inability of the heart to provide adequate circulation for the body's needs or only able to do so at increased cardiac filling pressures. It is the cardiovascular syndrome projected to increase most [4] in the USA with the largest attendant loss of productivity and increase in direct medical cost in the next 20 years [4]. Europe and Asia show similar data [5, 6], with a rapid increase in disease burden on society. Diagnosis is difficult and the prognosis in HF remains poor. Evidence-based therapy of proven efficacy has been introduced over the last 25 years but mortality and readmission rates remain high.

\section{Plasma extracellular vesicles}

Extracellular vesicles, including microvesicles, microparticles and exosomes [7], are abundant in plasma. Extracellular vesicles are secreted by all cells, especially after stimulation of inflammatory cells. These vesicles are important in cell to cell communication in a variety of processes including coagulation [8], antigen presentation [9] and tissue damage [10]. Plasma extracellular vesicles can be easily isolated from frozen plasma or serum and contain protein, miRNA and RNA depending on source and stimulus.

Extracellular vesicles that play a role in the initiation of thrombus formation are of major interest in cardiovascular disease pathogenesis, since thrombosis is the major underlying cause of cardiovascular events. In this process, monocytederived extracellular vesicles play an important role. Tissue factor (TF), as an important initiator of thrombosis, was found in vesicles that are positive for the monocyte marker CD14
(Fig. 1). In vitro studies using endothelial cells (EC) showed that these TF-containing vesicles do not only initiate thrombosis but also adhere to the EC that results in increased EC thrombogenicity via elevation of TF activity in the $\mathrm{EC}$ and decrease of tissue factor pathway inhibitor and thrombomodulin [11]. This procoagulant activity, with monocyte-derived circulating TF as its main source, was associated with coronary artery disease [12] and in stent thrombosis [13].

If extracellular vesicle proteins are involved in cardiovascular events, then plasma vesicle protein content might be an important biomarker source for prognosis and prediction of cardiovascular events.

\section{Extracellular vesicle proteins for diagnosis of ACS}

Circulating miRNAs are, at least partly, derived from extracellular vesicles and have been used in the diagnosis of acute coronary syndrome (ACS, [14]). Extracellular vesicle protein levels, however, have only recently been described to carry information in the presence of ACS [15]. Following a proteomics discovery approach, we demonstrated in a chest pain cohort of 471 patients that pIgR, Cystatin C (CST3), C5a and total vesicle protein concentration are associated with the presence of ACS. Comparisons of this association between male and female patients reveal these markers to be highly associated with ACS in males but not with ACS in females. This striking difference is in agreement with an increasing body of evidence that women tend to have lower rates of coronary artery occlusions and have a more preserved left

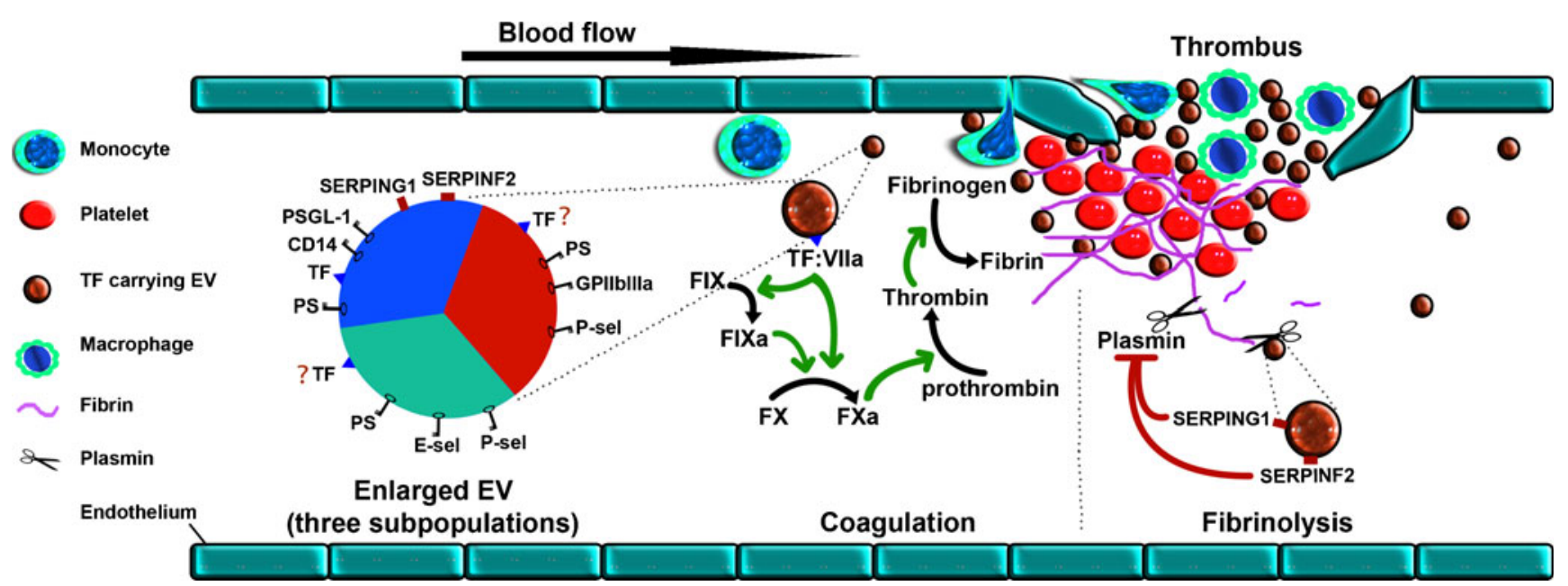

Fig. 1 Diagram showing an overview of the potential plasma extracellular vesicle procoagulant activity. Subpopulations of extracellular vesicles (EVs) in the plasma involved in coagulation are thought to have three origins: monocyte-derived EVs (blue), platelet-derived EVs (red) and endothelial cell-derived EVs (green) as outlined in the enlarged EV 3colour pie chart. EVs contain proteins involved in coagulation and fibrinolysis. For coagulation TF is the key initiator of the coagulation cascade and forms a complex with factor VIIa (FVIIa). This complex activates factor IX (FIX) and factor X (FX). The activated factor X (FXa) further cleaves prothrombin into thrombin that converts fibrinogen into fibrin to form clot. For fibrinolysis, the plasmin inhibitors SERPING1 and SERPINF2 have been identified in EVs and inhibit the breakdown of a fibrin via fibrinolysis. Abbreviations: EV, extracellular vesicle; TF, tissue factor; PSGL-1, P-selectin glycoprotein ligand-1; PS, phosphatidylserine; P-sel, P-selectin; E-sel, E-selectin 
ventricular function. Women, however, also have a higher mortality and a worse prognosis following MI compared with men. The established difference in extracellular vesicle protein levels associated with ACS identifies vesicles as an excellent biomarker source for gender-specific diagnosis and prognosis of ACS. Furthermore, this might help in understanding the underlying gender differences in the pathophysiology of ACS.

\section{Extracellular vesicle proteins for prognosis of secondary cardiovascular events}

The number of survivors of a first cardiovascular event is on the rise globally with a rapid increase in Asia. These patients are at high risk for a second cardiovascular event even after adequate control of risk factors. The number and type of extracellular vesicles that are $\mathrm{CD} 31^{+} / \mathrm{Annexin} \mathrm{V}^{+}$positive were associated with an increased risk for secondary events consisting of myocardial infarction, percutaneous coronary intervention (PCI), coronary artery bypass graft (CABG), stroke and vascular mortality [16].

For the first time, the protein content of all plasma extracellular vesicles and its association with secondary cardiovascular events were studied in a large clinical cohort [17].

Comparing plasma extracellular vesicles of patients with a secondary cardiovascular event to gender- and age-matched patients without a second cardiovascular event, we identified four vesicle proteins that were different between the two groups. These vesicle proteins were Cystatin C, SerpinG1, SerpinF2 and CD14 and were validated in the SMART cohort. This revealed that three of these vesicle proteins (Cystatin C, SerpinF2 and CD14) were associated with future cardiovascular events after correction of risk factors such as age, gender, smoking and eGFR.

For this, measurement of plasma extracellular vesicle protein levels has the potential to stratify patients at risk for secondary events on top of risk factors.
There is much uncertainty over the relative contribution of platelets to the overall circulating extracellular vesicle population. Platelets are a key intermediary in the blood extracellular vesicle landscape because their function is readily and rapidly modified by platelet-directed medications. The ongoing Stratifying risk with Metabolomics And platelet Reactivity Testing in Acute Coronary Syndrome (SMART-ACS) study, initiated in Singapore, includes platelet reactivity testing of more than 1000 patients undergoing invasive management of ACS, using impedance aggregometry (multiple electrode aggregometry, Roche Diagnostics, Indianapolis, IN) and follow up for secondary cardiovascular events. This will allow us to investigate how platelet-directed medications may potentially modify the content of the plasma extracellular vesicle population and its association with secondary events.

\section{Caucasian and Asian cardiovascular biobanking}

The identification and validation of new biomarkers is highly dependent on the availability of patient cohorts with detailed phenotypic characterisation. Important ethnic differences have been described in the susceptibility, risk factors, and outcomes with cardiovascular disease. For example: Indian men in the UK have an adjusted hazard ratio of 1.45 (CI: 1.29 to 1.63 ) for cardiovascular disease while Chinese men in the UK have an adjusted hazard ratio of 0.51 (CI: 0.32 to 0.83 ) compared with Caucasian men [18]. Among Southeast Asian patients with HF in Singapore, Indians and Malays have been shown to suffer worse outcomes than Chinese [19] although the reasons remain unclear [20]. The ADHERE-Asia Pacific Registry showed that compared with other multicentre registries in the US and Europe, Asian patients hospitalised with HF were younger and had more severe clinical features.

For this, we initiated the (ongoing) biobanking efforts in both Singapore and the Netherlands (Fig. 2). In 2008, the
Fig. 2 Overview of ongoing cardiovascular biobanking in Singapore and the Netherlands. Red boxes indicate matched biobank studies, green boxes indicate work in progress of matching Singapore and Dutch biobank studies, numbers indicate patients included $\sim$ June 2013
NL (Caucasian)

Atherectomy $3000+$ pts

Bypass $400+$ pts

$\mathrm{PCl} 1500+$ pts

Chest-pain $1000+$

Heart failure $500+$
SG (Chinese, Malay, Indian)

Atherectomy $100+$ pts

Bypass 1500+pts

$\mathrm{PCl} 1500+$ pts 
design of the AtheroExpress [21] collecting tissue, plasma and blood cells with a follow-up for secondary cardiovascular events was used to start up the Singapore AtheroExpress at the National University Heart Center Singapore (NUHCS). This Singapore biobank is collecting, besides atherectomy samples, also aortic punctures during CABG next to plasma and blood cells, and follows patients up for secondary cardiovascular events. Aortic punctures and plasma are now also collected at the UMC Utrecht in Pegasus using the same design.

Singapore initiated the collection of plasma of patients undergoing a PCI with follow-up for secondary cardiovascular events. A year later, the UMC Utrecht 'mirror' biobank of PCI patients was initiated, a collective effort that now includes over 1000 patients a year.

Work is in progress to match patients with chest pain in the emergency department of the Meander Hospital in Amersfoort (Minerva biobank cohort) with chest pain cohorts at NUHCS and Singapore General Hospital as well as the extensive HF cohort initiatives in Singapore [22] and Asia [23] with Dutch heart failure cohorts.

A key advantage of these mirrored cohorts is the implementation of common data and tissue collection and storage protocols across sites in both countries. This facilitates comparisons of biomarker profiles between Dutch and Singapore populations; such an effort is currently ongoing, with measurement of high sensitivity troponin $\mathrm{T}$, high sensitivity C-reactive protein, von Willebrand factor, myeloperoxidase and Cystatin $\mathrm{C}$ in 1200 Dutch and 1000 Singaporean patients who have undergone elective coronary angiography.

\section{Future perspectives}

Plasma extracellular vesicle proteins are still in the early stage of study, mostly aiming to identify and to characterise the proteomes. Although relatively unexplored due to technical challenges, vesicle proteins hold promise as mass spectrometrybased proteomic technology is rapidly emerging for identification and quantification of extremely low abundant proteins in a sample. This has already resulted in identification of vesicle proteins for the diagnosis of ACS [15] and prognosis of secondary cardiovascular events [17] in clinical cohorts. Isolation of plasma extracellular vesicle subpopulations is expected to lead to new and better cardiovascular vesicle protein biomarkers in a new sample set.

This rapidly growing vesicle technology will hold, in combination with the maturation of the biobanks (inclusion of patients and secondary events) in the Netherlands and Asia, great promise for new diagnostic and prognostic biomarkers that take ethnicity and gender into account.
Acknowledgements We would like to thank the Strategic fund of the Royal Netherlands Academy of Arts and Sciences for supporting this study.

Conflicts of interest None reported.

Open Access This article is distributed under the terms of the Creative Commons Attribution License which permits any use, distribution, and reproduction in any medium, provided the original author(s) and the source are credited.

\section{References}

1. Preventing chronic disease: a vital investment. Geneva: WHO; 2005.

2. The WHO Report 2002: reducing risks, promoting healthy life. Geneva: WHO; 2002.

3. Ueshima H, Sekikawa A, Miura K, et al. Cardiovascular disease and risk factors in Asia: a selected review. Circulation. 2008;118:2702-9.

4. Heidenreich PA, Trogdon JG, Khavjou OA, et al. Forecasting the future of cardiovascular disease in the United States: a policy statement from the American Heart Association. Circulation. 2011;123(8):93344.

5. Stewart S. Financial aspects of heart failure programs of care. Eur J Heart Fail. 2005;7(3):423-8.

6. Leong KT, Goh PP, Chang BC, Lingamanaicker J. Heart failure cohort in Singapore with defined criteria: clinical characteristics and prognosis in a multi-ethnic hospital-based cohort in Singapore. Singapore Med J. 2007;48(5):408-14.

7. Tushuisen Diamant M, Sturk A, Nieuwland R. Cell-derived microparticles in the pathogenesis of cardiovascular disease: friend or foe? Arterioscler Thromb Vasc Biol. 2011;31:4-9.

8. Del Conde I, Shrimpton CN, Thiagarajan P, López JA. Tissuefactor-bearing microvesicles arise from lipid rafts and fuse with activated platelets to initiate coagulation. Blood. 2005;106(5):1604 11.

9. Théry C, Ostrowski M, Segura E. Membrane vesicles as conveyors of immune responses. Nat Rev Immunol. 2009;9(8):581-93.

10. Lai RC, Arslan F, Lee MM, et al. Exosome secreted by MSC reduces myocardial ischemia/reperfusion injury. Stem Cell Res. 2010;4:214 22.

11. Aharon A, Tamari T, Brenner B. Monocyte-derived microparticles and exosomes induce procoagulant and apoptotic effects on endothelial cells. Thromb Haemost. 2008;100:878-85.

12. Leatham EW, Bath PMW, Tooze A. Increased monocyte tissue factor expression in coronary disease. Br Heart J. 1995;73:10-3.

13. Palmerini T, Coller BS, Cervi V, et al. Monocyte-derived tissue factor contributes to stent thrombosis in an in vitro system. J Am Coll Cardiol. 2004;44:1570-7.

14. Oerlemans MI, Mosterd A, Dekker MS, et al. Early assessment of acute coronary syndromes in the emergency department: the potential diagnostic value of circulating microRNAs. EMBO Mol Med. 2012; 4(11):1176-85.

15. de Hoog VC, Timmers L, Schoneveld AH, et al. Serum extracellular vesicle protein levels are associated with acute coronary syndrome. Eur Heart J: Acute Cardiov Care. 2013;2:53-60.

16. Sinning JM, Losch J, Walenta K, Böhm M, Nickenig G, Werner N. Circulating CD31+/Annexin V + microparticles correlate with cardiovascular outcomes. Eur Heart J. 2011;32:2034-41.

17. Kanhai DA, Visseren FLJ, Van der Graaf Y, et al. Microvesicle protein levels are associated with increased risk for future vascular 
events and mortality in patients with clinically manifest vascular disease. Int J Cardiol. 2013; Feb 25.

18. Hippisley-Cox J, Coupland C, Vinogradova Y, et al. Predicting cardiovascular risk in England and Wales: prospective derivation and validation of QRISK2. BMJ. 2008;336(7659):1475-82.

19. Stewart S, MacIntyre K, Capewell S, McMurray JJV. An ageing population and heart failure: an increasing burden in the 21st Century? Heart. 2003;89:49-53.

20. Lee R, Chan S-P, Wong J, Lau D, Ho K-T, Ng K. Impact of diabetes mellitus on survival in South East Asian patients with congestive heart failure due to left ventricular systolic dysfunction. Int J Cardiol. 2010;142:97-1002.
21. Verhoeven BA, Velema E, Schoneveld AH, et al. Athero-express: differential atherosclerotic plaque expression of mRNA and protein in relation to cardiovascular events and patient characteristics. Rationale and design. Eur J Epidemiol. 2004;19(12):1127-33.

22. Santhanakrishnan R, Ng TP, Cameron VA, et al. The Singapore heart failure outcomes and phenotypes (SHOP) study and prospective evaluation of outcome in patients with heart failure with preserved left ventricular ejection fraction (PEOPLE) study: rationale and design. J Card Fail. 2013;19(3):156-62.

23. Lam CS, Anand I, Zhang S, et al. Asian sudden cardiac death in heart failure (ASIAN-HF) registry. Eur J Heart Fail. 2013; Apr 7. [Epub ahead of print] PubMed PMID: 23568645. 